\title{
EXISTENCE FOR A FOURTH-ORDER BOUNDARY VALUE PROBLEM UNDER A TWO-PARAMETER NONRESONANCE CONDITION
}

\author{
MANUEL A. DEL PINO AND RAÚL F. MANÁSEVICH
}

(Communicated by Kenneth R. Meyer)

\begin{abstract}
We show existence for a nonlinear fourth-order boundary value problem under a nonresonance condition involving a two-parameter linear eigenvalue problem. We also state extensions of this result to certain higher-order P.D.E. cases.
\end{abstract}

\section{INTRODUCTION}

In this paper we are mainly concerned with existence for a fourth-order boundary value problem of the form

$$
\begin{gathered}
y^{(I V)}=f\left(x, y, y^{\prime \prime}\right) \quad 0<x<1 \\
y(0)=y_{0}, y(1)=y_{1}, \quad y^{\prime \prime}(0)=\bar{y}_{0}, \quad y^{\prime \prime}(1)=\bar{y}_{1}
\end{gathered}
$$

where $f:[0,1] \times \mathbb{R} \times \mathbb{R} \rightarrow \mathbb{R}$ is continuous. Our aim is to establish additional conditions on $f$ which ensure the solvability of (1.1)-(1.2) for any $y_{0}, y_{1}$, $\bar{y}_{0}, \bar{y}_{1}$.

In [1], Aftabizadeh showed the existence of a solution to (1.1)-(1.2) under the restriction that $f$ is a bounded function. In [ 3, Theorem 1], Yang extended this existence result, letting $f$ satisfy a growth condition of the form

$$
|f(x, y, z)| \leq a|y|+b|z|+c
$$

for all $x \in[0,1]$. Here, $a, b$, and $c$ are positive constants such that

$$
\frac{a}{\pi^{4}}+\frac{b}{\pi^{2}}<1 \text {. }
$$

Yang's result also extended a previous work of Usmani [2], who considered an $f$ of the form $f\left(x, y, y^{\prime \prime}\right)=g(x) y+h(x)$ with $\sup _{x \in[0,1]}|g(x)|<\pi^{4}$.

Received by the editors September 19, 1989.

1980 Mathematics Subject Classification (1985 Revision). Primary 34B15; Secondary 34B10.

Key words and phrases. Nonresonance, two-parameter eigenvalue problem, Leray-SchauderTheorem.

This research was sponsored by FONDECYT, research grant $0546-88$, and by the DTI. U. de Chile. 
Yang further proved the unique solvability of (1.1)-(1.2) for $f$ of such form, but with $g$ satisfying the nonresonance condition

$$
(k \pi)^{4}<g(x)<((k+1) \pi)^{4}
$$

for some $k \in \mathbb{N}$ and for all $x \in[0,1]$.

We note here that the sequence $\left\{(k \pi)^{4}\right\}_{k=1}^{\infty}$ corresponds to the eigenvalues of the problem

$$
\begin{gathered}
y^{(I V)}=\lambda y, \\
y(0)=0=y(1)=y^{\prime \prime}(0)=y^{\prime \prime}(1) .
\end{gathered}
$$

In $\S 2$ of this paper we study the two-parameter eigenvalue problem

$$
\begin{gathered}
y^{(I V)}=\alpha y-\beta y^{\prime \prime}, \\
y(0)=0=y(1)=y^{\prime \prime}(0)=y^{\prime \prime}(1),
\end{gathered}
$$

which generalizes (1.6)-(1.7). In $\S 3$, using the results of $\S 2$ and a more general condition than that given by (1.3)-(1.4) we considerably extend the abovementioned results.

Our results can also be generalized to higher-order equations, as well as to some semilinear elliptic problems. In this direction, in $\S 4$ we show one possible generalization.

\section{Preliminary Results}

We begin this section by solving the eigenvalue problem (1.8)-(1.9). A pair $(\alpha, \beta)$ such that $(1.8)-(1.9)$ possesses a nontrivial solution will be called an eigenvalue pair. A corresponding nontrivial solution will be called an eigenfunction.

Proposition 2.1. $(\alpha, \beta)$ is an eigenvalue pair of (1.8)-(1.9) (if and only if)

$$
\frac{\alpha}{(k \pi)^{4}}+\frac{\beta}{(k \pi)^{2}}=1
$$

for some $k \in \mathbb{N}$.

Proof. Let $L y=y^{\prime \prime}$. Then

$$
y^{(I V)}+\beta y^{\prime \prime}-\alpha y=\left(L+r_{1}\right)\left(L+r_{2}\right) y
$$

for some $r_{1}, r_{2} \in \mathbb{C}$. It is easy to see that if $(1.8)-(1.9)$ possesses a nontrivial solution, then either $r_{1}=(k \pi)^{2}$ or $r_{2}=(k \pi)^{2}$ for some $k \in \mathbb{N}$. In either case, $\sin k \pi x$ is a nontrivial solution of (1.8)-(1.9). By substituting this solution into (1.8), (2.1) follows. Reciprocally, if (2.1) holds, then clearly $\sin k \pi x$ is a nontrivial solution of (1.8)-(1.9).

Next, for $j \in \mathbb{N}$, let us set

$$
L_{j}=\left\{(\alpha, \beta) \mid \frac{\alpha}{(j \pi)^{4}}+\frac{\beta}{(j \pi)^{2}}=1\right\} .
$$


In view of the above proposition, we call $L_{j}$ an eigenline of (1.8)-(1.9). We note that an eigenvalue pair $(\alpha, \beta)$ can belong to at most two eigenlines. If $(\alpha, \beta)$ belongs to just one $L_{j}$, then the corresponding eigenspace is that spanned by $\sin j \pi x$. If $(\alpha, \beta)$ belongs to $L_{j} \cap L_{k}$ then the corresponding eigenspace is that spanned by $\sin j \pi x$ and $\sin k \pi x$.

Suppose now that the pair $(\alpha, \beta)$ is not an eigenvalue pair of $(1.8)-(1.9)$; i.e.,

$$
\frac{\alpha}{(k \pi)^{4}}+\frac{\beta}{(k \pi)^{2}} \neq 1
$$

for all $k \in \mathbb{N}$ and that $h \in L^{2}(0,1)$. From, for example, the Fredholm Alternative, it follows that the boundary value problem

$$
\begin{gathered}
y^{(I V)}=\alpha y-\beta y^{\prime \prime}+h(x), \\
y(0)=0=y(1)=y^{\prime \prime}(0)=y^{\prime \prime}(1)
\end{gathered}
$$

has a unique solution for each $h \in L^{2}(0,1)$. Moreover, this solution admits a Fourier series expansion of the form

$$
y(x)=\sum_{k=1}^{\infty} \frac{h_{k} \sin k \pi x}{k^{4} \pi^{4}-\alpha-\beta k^{2} \pi^{2}},
$$

where

$$
h(x)=\sum_{k=1}^{\infty} h_{k} \sin k \pi x
$$

Also, we have that

$$
y^{\prime \prime}(x)=-\sum_{k=1}^{\infty} \frac{k^{2} \pi^{2} h_{k} \sin k \pi x}{k^{4} \pi^{4}-\alpha-\beta k^{2} \pi^{2}} .
$$

From (2.8) and (2.9) we can easily see that the operators $A, B: L^{2}(0,1) \rightarrow$ $L^{2}(0,1)$ defined by,

$$
A(h)=y, \quad B(h)=y^{\prime \prime}
$$

are compact linear operators. In (2.10), $y$ is the solution of $(2.5)-(2.6)$ corresponding to $h \in L^{2}(0,1)$. The norms of $A$ and $B$ are respectively given by

$$
\|A\|=\max _{k \in \mathbb{N}}\left\{\frac{1}{\left|k^{4} \pi^{4}-\alpha-\beta k^{2} \pi^{2}\right|}\right\},\|B\|=\max _{k \in \mathbb{N}}\left\{\frac{k^{2} \pi^{2}}{\left|k^{4} \pi^{4}-\alpha-\beta k^{2} \pi^{2}\right|}\right\} .
$$




\section{EXISTENCE UNDER A NONRESONANCE CONDITION}

In this section we state and prove our main result.

Theorem 3.1. Assume that the pair $(\alpha, \beta)$ satisfies

$$
\frac{\alpha}{(k \pi)^{4}}+\frac{\beta}{(k \pi)^{2}} \neq 1 \text {, }
$$

for all $k \in \mathbb{N}$, and that there are positive constants $a, b$, and $c$ such that

$$
a \max _{k \in \mathbb{N}}\left\{\frac{1}{\left|k^{4} \pi^{4}-\alpha-\beta k^{2} \pi^{2}\right|}\right\}+b \max _{k \in \mathbb{N}}\left\{\frac{k^{2} \pi^{2}}{\left|k^{4} \pi^{4}-\alpha-\beta k^{2} \pi^{2}\right|}\right\}<1 .
$$

and

$$
|f(x, y, z)-(\alpha y-\beta z)| \leq a|y|+b|z|+c
$$

for all $x \in[0,1], y \in \mathbb{R}, z \in \mathbb{R}$ then problem (1.1)-(1.2) possesses at least one solution.

Before going into the details of the proof, we first note that Theorem 1 of [3] follows from Theorem 3.1 by just setting $(\alpha, \beta)=(0,0)$. Also, we note that (3.1)-(3.2) trivially implies that

$$
\frac{a}{\left|k^{4} \pi^{4}-\alpha-\beta k^{2} \pi^{2}\right|}+\frac{b k^{2} \pi^{2}}{\left|k^{4} \pi^{4}-\alpha-\beta k^{2} \pi^{2}\right|}<1
$$

for all $k \in \mathbb{N}$. It turns out that (3.4) is equivalent to the fact that the square $[\alpha-a, \alpha+a] \times[\beta-b, \beta+b]$ does not intersect any of the eigenlines $L_{j}$ of (1.8)-(1.9). From this point of view (3.1)-(3.2) can be thought of as a twoparameter nonresonance condition relative to the eigenlines $L_{j}$. In particular, in the case of $f$ of class $C^{1}$, what Theorem 3.1 says is roughly that if for large $|y|+|z|$ the pair

$$
\left(\frac{\partial f}{\partial y}, \frac{\partial f}{\partial z}\right)
$$

lies on a certain closed rectangle in $\mathbb{R}^{2}$ which does not intersect the eigenlines $L_{j}, j \in \mathbb{N}$, of $(1.8)-(1.9)$, then problem (1.1)-(1.2) is solvable. Finally, we conjecture that (3.4) instead of (3.2) suffices for the validity of Theorem 3.1.

Proof of Theorem 3.1. Without loss of generality, we can suppose $y_{0}=0=y_{1}=$ $\bar{y}_{0}=\bar{y}_{1}$ in (1.2). Let us define $T: L^{2}(0,1) \times L^{2}(0,1) \rightarrow L^{2}(0,1) \times L^{2}(0,1)$ by

$$
T(y, z)=(A(f(\cdot, y, z)-(\alpha y-\beta z)), B(f(\cdot, y, z)-(\alpha y-\beta z))),
$$

where $A$ and $B$ are the operators defined in (2.10). The growth condition (3.3) together with the compactness of $A$ and $B$ imply that $T$ is a completely continuous operator. Also, it is clear that $(1.1)-(1.2)$ is equivalent to the fixed point problem in $L^{2}(0,1) \times L^{2}(0,1)$

$$
(y, z)=T(y, z) .
$$


We will study this fixed-point problem by means of the well known LeraySchauder Theorem. To do this, we show that there is a uniform bound independent of $t \in[0,1]$ for the solutions of the equation

$$
(y, z)=t T(y, z) \text {. }
$$

Thus let $(y, z)$ be a solution of (3.7). From the definition of $T$ and (3.3), we obtain the result that

$$
\|y\|_{L^{2}} \leq\|A\|\left\{a\|y\|_{L^{2}}+b\|z\|_{L^{2}}+c\right\}
$$

and

$$
\|z\|_{L^{2}} \leq\|B\|\left\{a\|y\|_{L^{2}}+b\|z\|_{L^{2}}+c\right\} .
$$

By combining (3.8) and (3.9) and using (3.2) and (2.11), we obtain the existence of a constant $K=K(a, b, c,\|A \mid\| B \|$,$) such that$

$$
\|y\|_{L^{2}}+\|z\|_{L^{2}} \leq K \text {. }
$$

From the Leray-Schauder Theorem we conclude the existence of at least one solution to (3.6), and the theorem follows.

Remark. The uniqueness of the solution predicted by Theorem 3.1 can be easily shown if we replace (3.3) by the stronger Lipschitz-type condition

$$
\mid(f(x, y, z)-(\alpha y-\beta z)-f(x, \bar{y}, \bar{z})-(\alpha \bar{y}-\beta \bar{z}) \leq a|y-\bar{y}|+b|z-\bar{z}| .
$$

In the case that $f(x, y, z)$ is of class $C^{1}$ in $(y, z),(3.11)$ is equivalent to

$$
\left|\frac{\partial f}{\partial y}(x, y, z)-\alpha\right| \leq a, \quad\left|\frac{\partial f}{\partial z}(x, y, z)-\beta\right| \leq b
$$

for every $x \in[0,1], y$ and $z$ in $\mathbb{R}$. For example, if

$$
f(x, y, z)=g(x) y+h(x),
$$

with $g$ and $h$ continuous in $[0,1]$, we recover the uniqueness assertion of Theorem 2 of [3] by setting $\beta=b=0$ in (3.12).

\section{AN EXTENSION OF THEOREM 3.1}

Theorem 3.1 can be generalized to some higher-order elliptic problems. We establish here one possible extension.

Let $\Omega$ be a bounded domain in $\mathbb{R}^{m}$ and $f: \Omega \times \mathbb{R}^{n} \rightarrow \mathbb{R}^{n}$ satisfy a Carathéodory condition. Consider the problem

$$
\begin{gathered}
(-\Delta)^{n} u=f\left(x, u,-\Delta u, \ldots,(-\Delta)^{n-1} u\right), \\
u,-\Delta u, \ldots,(-\Delta)^{n-1} u \in H_{0}^{1}(\Omega) .
\end{gathered}
$$

By a solution of (4.1)-(4.2), we understand a weak solution $\left(z_{0}, \ldots, z_{n-1}\right) \in$ $\left(H_{0}^{1}(\Omega)\right)^{n}$ of the system

$$
-\Delta z_{i-1}=z_{i}, \quad \text { for } i=1, \ldots, n-1
$$




$$
-\Delta z_{n-1}=f\left(x, z_{1}, \ldots, z_{n-1}\right) .
$$

Let $\left\{\lambda_{k}\right\}_{k=1}^{\infty}$ be the sequence of eigenvalues of $-\Delta$ under Dirichlet boundary conditions. We have the following existence result, whose proof can be carried out using the same arguments employed in proving Theorem 3.1.

Theorem 4.1. Assume that there are real numbers $\alpha_{0}, \alpha_{1}, \ldots, \alpha_{n-1}$, such that

$$
\frac{\alpha_{0}}{\lambda_{k}^{n}}+\frac{\alpha_{1}}{\lambda_{k}^{n-1}}+\cdots+\frac{\alpha_{n-1}}{\lambda_{k}} \neq 1 \text { for all } k \in \mathbb{N}
$$

and positive constants $a_{0}, a_{1}, \ldots, a_{n-1}, c$, such that

$$
\sum_{i=0}^{n-1} a_{i} \max _{k \in \mathbb{N}}\left\{\frac{\lambda_{k}^{i}}{\left|\lambda_{k}^{n}-\sum_{j=0}^{n-1} \alpha_{j} \lambda_{k}^{j}\right|}\right\}<1
$$

and such that

$$
\left|f\left(x, s_{0}, \ldots, s_{n-1}\right)-\sum_{i=0}^{n-1} \alpha_{i} s_{i}\right| \leq \sum_{i=0}^{n-1} a_{i}\left|s_{i}\right|+c,
$$

for all $x \in \Omega, s_{0}, \ldots, s_{n-1} \in \mathbb{R}$. Then (4.1)-(4.2) possesses at least one solution.

Uniqueness can be obtained replacing (4.7) by a Lipschitz condition similar to $(3.10)$.

\section{REFERENCES}

1. A. R. Aftadizadeh, Existence and uniqueness theorems for fourth-order boundary problems, J. Math. Anal. Appl. 116 (1986), 415-426.

2. R. A. Usmani, $A$ uniqueness theorem for a boundary value problem, Proc. Amer. Math. Soc. 77 (1979), 327-335.

3. Y. Yang, Fourth-order two-point boundary problems, Proc. Amer. Math. Soc. 104 (1988), $175-180$.

School of Mathematics, University of Minnesota, Minneapolis, Minnesota 55455

Departamento de Mathemáticas, F.C.F.M. Universidad de Chile, Casilla 170, Correo 3, Santiago, Chile 